\title{
Rickettsia and Borrelia Prevalence Study among Ticks in Georgia
}

\author{
E. Zghenti ${ }^{1}$, R. Sukhiashvili ${ }^{1}$, E. Khmaladze ${ }^{1}$, N. Tsertsvadze ${ }^{1}$, Sarah Pisarcik ${ }^{2}$ and P. \\ Imnadze ${ }^{1}$
}

${ }^{1}$ National Center for Disease Control and Public Health, Tbilisi, Georgia; ${ }^{2}$ USAMRIID, Frederick, MD, USA

\section{Objective}

The primary goal of this study was to assess the prevalence of Rickettsia and Borellia in ticks collected from different regions of Georgia.

\section{Introduction}

Rickettsiae are an important arthropod-borne pathogens widely distributed throughout the world that infects humans causing various types of clinical diseases. The clinical features of rickettsial diseases can often overlap with other tick-borne diseases, particularly with borrelioses. These organisms can share the same transmitting vector; co-infection in a single vector is possible, leading to further complication of the diagnosis and treatment of these diseases. Preliminary study showed a relatively high infection rate for spotted fever group Rickettsiae among ticks in Georgia. The prevalence of Borrelia in ticks has not been studied so far.

\section{Methods}

A total of 1000 single species vector pools, representing 23 tick species collected from 8 regions of Georgia were studied. Genus-specific qPCR assays have been validated and applied to detect $17-\mathrm{kDa}$ protein gene of Rickettsia species and 16S rRNA gene of Borrelia burgdorferi sensu lato. A database for all samples utilized in this study was created including information about tick species, sex, maturity, collection location, method of collection, time of day etc.. The minimum infection rates (MIR) were calculated for the pathogens in vectors and for geographical sites as well.

\section{Results}

Obtained results revealed that $33 \%$ of sample pools were positive on Rickettsia spp., $13 \%$ on Borrelia burgdorferi; $10 \%$ of the pools were positive for both Rickettsia spp., and Borrelia burgdoferi. Rickettsia was found in ticks from all 8 regions sampled, indicating the presence of Rickettsia throughout Georgia. The majority of the Rickettsia infected ticks were localized in the Samtskhe-Javakheti and Samegrelo-Zemo Svanti regions. Distribution of B. burgdorferi was not uniform. The majority of the $B$. burgdorferi infected ticks were localized in the Guria region.

Rickettsia spp., and B. burgdorferi were found in all tick species with the exception of ticks of the genus Argasidae. Ticks of the Hyalomma genus had the highest MIR for both Rickettsia and B. burgdorferi. For Rickettsia, Dermacenter marginatus showed the highest MIR of the ticks analyzed in this study; the highest MIR for B. burgdorferi was observed in $\mathrm{H}$. aegyptum.

\section{Conclusions}

Screening ticks for Rickettsia spp., and Borrelia burgdorferi has provided insights regarding the distributions and endemicity of potentially pathogenic and emerging tick-borne agents. Data obtained on the prevalence of these pathogens in ticks can be applied to assess the risk for human health of tick-borne diseases and to elicit tick related illnesses that may pose unidentified health risks. Continued study and monitoring will improve biosurveillance and play an important role in public health assessment for related disease risks.

\section{Keywords}

Rickettsia; Georgia; Borrelia

\section{Acknowledgments}

Defense Threat Reduction Agency, Cooperative Biological Engagement Program

\section{* Richard Obiso}

E-mail: rik@attimogroup.com 